\title{
The Mean Square of Divisor Function
}

\author{
Chaohua Jia and Ayyadurai Sankaranarayanan
}

Abstract. Let $d(n)$ be the divisor function. In 1916, S. Ramanujan stated but
without proof that
\[ \sum_{n \leq x} d^{2}(n)=x P(\log x)+E(x) \]

where $P(y)$ is a cubic polynomial in $y$ and

$$
E(x)=O\left(x^{\frac{3}{5}+\varepsilon}\right),
$$

where $\varepsilon$ is a sufficiently small positive constant. He also stated that, assuming the Riemann Hypothesis(RH),

$$
E(x)=O\left(x^{\frac{1}{2}+\varepsilon}\right) .
$$

In 1922, B. M. Wilson proved the above result unconditionally. The direct application of the $\mathrm{RH}$ would produce

$$
E(x)=O\left(x^{\frac{1}{2}}(\log x)^{5} \log \log x\right) .
$$

In 2003, K. Ramachandra and A. Sankaranarayanan proved the above result without any assumption.

In this paper, we shall prove

$$
E(x)=O\left(x^{\frac{1}{2}}(\log x)^{5}\right) .
$$

\section{Introduction}

Let $d(n)$ be the divisor function. In 1916, S. Ramanujan[9] stated but without proof that

$$
\begin{aligned}
& d^{2}(1)+d^{2}(2)+d^{2}(3)+\cdots+d^{2}(n) \\
& =A n(\log n)^{3}+B n(\log n)^{2}+C n \log n+D n+O\left(n^{\frac{3}{5}+\varepsilon}\right)
\end{aligned}
$$

here

2010 Mathematics Subject Classification: Primary 11M; Secondary 11M06.

Key words and phrases: divisor function, Riemann zeta-function, mean value. 


$$
A=\frac{1}{\pi^{2}}, \quad B=\frac{12 \gamma-3}{\pi^{2}}-\frac{36}{\pi^{4}} \zeta^{\prime}(2),
$$

where $\gamma$ is Euler's constant, $C, D$ are more complicated constants, $\varepsilon$ is a sufficiently small positive constant. S. Ramanujan[9] also stated that, assuming the Riemann Hypothesis( $\mathrm{RH})$, the error term in (1.1) can be improved to $O\left(n^{\frac{1}{2}+\varepsilon}\right)$.

Write

$$
E(x)=\sum_{n \leq x} d^{2}(n)-x P(\log x)
$$

where

$$
P(x)=A x^{3}+B x^{2}+C x+D .
$$

Then the statement of Ramanujan is that

$$
E(x)=O\left(x^{\frac{3}{5}+\varepsilon}\right)
$$

and assuming the $\mathrm{RH}$,

$$
E(x)=O\left(x^{\frac{1}{2}+\varepsilon}\right) .
$$

In 1922, B. M. Wilson[13] proved (1.4) unconditionally. By a general theorem of M. Kühleitner and W. G. Nowak(see (5.4) in [5]), we know

$$
E(x)=\Omega\left(x^{\frac{3}{8}}\right) .
$$

Let $d_{4}(n)$ be the general divisor function which is the number of representations of $n=d_{1} d_{2} d_{3} d_{4}$. In 1973, assuming

$$
\sum_{n \leq x} d_{4}(n)=\frac{1}{6} x \log ^{3} x+\left(2 \gamma-\frac{1}{2}\right) x \log ^{2} x+a x \log x+b x+O\left(x^{\alpha}\right),
$$

where $\gamma$ is Euler's constant, $a, b$ are constants, $\alpha$ is a constant strictly less than $\frac{1}{2}$, D. Suryanarayana and R. Sitaramachandra Rao[10] proved

$$
E(x)=O\left(x^{\frac{1}{2}} \exp \left(-c(\log x)^{\frac{3}{5}}(\log \log x)^{-\frac{1}{5}}\right)\right),
$$

where $c$ is a positive constant.

By Vinogradov's estimate, if $\frac{T}{2} \leq t \leq T$, then

$$
\frac{1}{\zeta(1+2 i t)} \ll(\log T)^{\frac{2}{3}}(\log \log T)^{\frac{1}{3}} .
$$


So it is not difficult to prove

$$
E(x)=O\left(x^{\frac{1}{2}}(\log x)^{\frac{17}{3}}(\log \log x)^{\frac{1}{3}}\right) .
$$

The direct application of the $\mathrm{RH}$ (or even the quasi-RH) would produce

$$
E(x)=O\left(x^{\frac{1}{2}}(\log x)^{5} \log \log x\right) .
$$

In 2003, K. Ramachandra and A. Sankaranarayanan[8] proved (1.8) without any assumption and put forward the following conjecture.

Conjecture(Ramachandra-Sankaranarayanan). Assuming the RH, we have

$$
E(x)=O\left(x^{\frac{1}{2}}\right) .
$$

For the average situation, in 2005, H. Maier and A. Sankaranarayanan[7] proved,

$$
\frac{1}{X} \int_{X}^{2 X} E^{2}(x) d x \ll X \exp \left(-c(\log X)^{\frac{3}{5}}(\log \log X)^{-\frac{1}{5}}\right),
$$

where $c$ is a positive constant.

In this paper, we shall prove the following theorem.

Theorem. If $E(x)$ is defined in (1.2), then unconditionally we have

$$
E(x)=O\left(x^{\frac{1}{2}}(\log x)^{5}\right) .
$$

Throughout this paper, we assume that $\varepsilon$ is a sufficiently small positive constant and that $T$ is sufficiently large.

\section{Some lemmas}

Lemma 1(Borel-Carathéodory). Suppose that $f(z)$ is holomorphic in the disk $\left|z-z_{0}\right| \leq R$ and that in the circle $z=z_{0}+\operatorname{Re}^{i \theta}(0 \leq \theta \leq 2 \pi)$,

$$
\operatorname{Re} f(z) \leq M
$$

Then in the disk $\left|z-z_{0}\right| \leq r(<R)$, we have

$$
|f(z)| \leq \frac{2 r}{R-r} M+\frac{R+r}{R-r}\left|f\left(z_{0}\right)\right| .
$$

See Section 5.5 of [11]. 
Lemma 2(Hadamard). Suppose that $f(z)$ is holomorphic in the disk $\left|z-z_{0}\right| \leq R_{3}, R_{1}<R_{2}<R_{3}$. Write

$$
M_{j}=\max _{\left|z-z_{0}\right|=R_{j}}|f(z)|, \quad j=1,2,3 .
$$

Then we have

$$
\log M_{2} \leq \frac{\log \left(\frac{R_{3}}{R_{2}}\right)}{\log \left(\frac{R_{3}}{R_{1}}\right)} \cdot \log M_{1}+\frac{\log \left(\frac{R_{2}}{R_{1}}\right)}{\log \left(\frac{R_{3}}{R_{1}}\right)} \cdot \log M_{3} .
$$

See Section 5.3 of [11].

Lemma 3. For $\alpha>0$ and $x>0$, we have

$$
\frac{1}{2 \pi i} \int_{\alpha-i \infty}^{\alpha+i \infty} \Gamma(s) x^{-s} d s=e^{-x}
$$

See (2.15.2) in page 33 of [12].

Lemma 4. For $-1 \leq \sigma \leq 2$ and $|t| \geq 1$, we have

$$
\Gamma(\sigma+i t) \ll|t|^{\sigma-\frac{1}{2}} e^{-\frac{\pi}{2}|t|} .
$$

See (4.12.2) in page 78 of [12].

Lemma 5. For $\operatorname{Re}(s)>1$, let

$$
f(s)=\sum_{n=1}^{\infty} \frac{a(n)}{n^{s}},
$$

where $a(n)=O(\psi(n)), \psi(n)$ is non-decreasing, and as $\sigma \rightarrow 1^{+}$,

$$
\sum_{n=1}^{\infty} \frac{|a(n)|}{n^{\sigma}}=O\left(\frac{1}{(\sigma-1)^{\alpha}}\right) .
$$

Then if $c>1, x$ is not an integer, and $N$ is the integer nearest to $x$,

$$
\begin{aligned}
\sum_{n<x} a(n)= & \frac{1}{2 \pi i} \int_{c-i T}^{c+i T} f(s) \frac{x^{s}}{s} d s+O\left(\frac{x^{c}}{T(c-1)^{\alpha}}\right) \\
& +O\left(\frac{\psi(2 x) x \log x}{T}\right)+O\left(\frac{\psi(N) x}{T|x-N|}\right) .
\end{aligned}
$$

See Lemma 3.12 in page 60 of [12].

Lemma 6. For $\operatorname{Re}(s)>1$, we have

$$
\sum_{n=1}^{\infty} \frac{d^{2}(n)}{n^{s}}=\frac{\zeta^{4}(s)}{\zeta(2 s)}
$$


See (1.2.10) in page 5 of [12].

Lemma 7. For $\operatorname{Re}(s) \geq \frac{1}{2}$ and $|s-1|>1$, we have

$$
\zeta(s)=O(|s|) .
$$

See (2.12.2) in page 29 of [12].

Lemma 8. For $\sigma \geq 1$ and $t \geq 1$, we have

$$
\frac{1}{\zeta(\sigma+i t)}=O(\log t)
$$

See (3.11.8) in page 60 of [12].

Lemma 9. For $t \geq 1$, we have

$$
\zeta\left(\frac{1}{2}+i t\right)=O\left(t^{\frac{1}{6}+\varepsilon}\right) .
$$

See Theorem 5.5 in page 99 of [12].

Remark. The bounds stated in Lemmas 8 and 9 suffice for our purpose though better upper bounds are known.

Lemma 10. For $\frac{1}{2} \leq \sigma \leq 1+\varepsilon$ and $t \geq 1$, we have

$$
\zeta(\sigma+i t)=O\left(t^{\frac{1}{3}(1-\sigma)+\varepsilon}\right) .
$$

It follows from Lemma 9 and the explanation in Chapter 5 of [12].

Lemma 11. We have

$$
\int_{1}^{T}\left|\zeta\left(\frac{1}{2}+i t\right)\right|^{4} d t=O\left(T \log ^{4} T\right) .
$$

See (7.6.1) in page 147 of [12].

Lemma 12(Huxley). For $\sigma \geq \frac{1}{2}$, let $N(\sigma, T, 2 T)$ denote the number of zeros $\rho=\beta+i \gamma$ of $\zeta(s)$ which satisfy $\beta \geq \sigma$ and $T \leq \gamma \leq 2 T$. Then

$$
N(\sigma, T, 2 T) \ll T^{\frac{12}{5}(1-\sigma)+\varepsilon} .
$$

See [3].

Lemma 13. For $\operatorname{Re}(z)>0$, we have

$$
\int_{0}^{\infty} e^{-z t}\left|\zeta\left(\frac{1}{2}+i t\right)\right|^{2} d t=2 \pi e^{\frac{i z}{2}} \sum_{l=1}^{\infty} d(l) \exp \left(2 \pi i l e^{i z}\right)+f(z),
$$

where $f(z)$ is holomorphic in $|z|<4 \varepsilon$. 
This is Lemma 1 in [4].

Define

$$
D\left(s ; \frac{h}{k}\right)=\sum_{l=1}^{\infty} \frac{d(l)}{l^{s}} e\left(l \frac{h}{k}\right) .
$$

Lemma 14(Estermann). Suppose that $(h, k)=1$. The function $D\left(s ; \frac{h}{k}\right)$ is meromorphic in the whole plane with only one pole of order 2 at $s=1$. In the neighborhood of $s=1$,

$$
D\left(s ; \frac{h}{k}\right)=\frac{1}{k} \cdot \frac{1}{(s-1)^{2}}+\frac{2}{k}(\gamma-\log k) \cdot \frac{1}{(s-1)}+\cdots,
$$

where $\gamma$ is Euler's constant. At $s=0$, we have

$$
D\left(0 ; \frac{h}{k}\right)=\frac{1}{4}-\frac{1}{\pi i} \sum_{a=1}^{k} \beta(a, k) \sum_{0<b<\frac{k}{2}} \eta(b, k) e\left(a b \frac{\bar{h}}{k}\right),
$$

where $\bar{h} h \equiv 1(\bmod k)$,

$$
\beta(a, k)=\left\{\begin{array}{cl}
\frac{1}{1-e\left(-\frac{a}{k}\right)}, & \text { if } 1 \leq a<k, \\
\frac{1}{2}, & \text { if } a=k,
\end{array}\right.
$$

and when $0<b<\frac{k}{2}$,

$$
0<\eta(b, k)<\frac{1}{b}
$$

Moreover, $D\left(s ; \frac{h}{k}\right)$ satisfies the functional equation

$$
D\left(s ; \frac{h}{k}\right)=2 G^{2}(s) k^{1-2 s}\left(D\left(1-s ; \frac{\bar{h}}{k}\right)-\cos (\pi s) D\left(1-s ;-\frac{\bar{h}}{k}\right)\right),
$$

where

$$
G(s)=(2 \pi)^{s-1} \Gamma(1-s) .
$$

See (21), (34), (32), (29) and (19) in [1].

Lemma 15. If $\left(m_{1}, m_{2}\right)=\left(n_{1}, n_{2}\right)=1$, then

$$
\left(m_{1} n_{1}^{2}, m_{2} n_{2}^{2}\right)=\left(m_{1}, n_{2}^{2}\right)\left(m_{2}, n_{1}^{2}\right)
$$

Proof. We have

$$
\left(m_{1} n_{1}^{2}, m_{2} n_{2}^{2}\right)=\left(m_{1}, n_{2}^{2}\right)\left(\frac{m_{1}}{\left(m_{1}, n_{2}^{2}\right)} n_{1}^{2}, m_{2} \frac{n_{2}^{2}}{\left(m_{1}, n_{2}^{2}\right)}\right) .
$$


Since

$$
\left(\frac{m_{1}}{\left(m_{1}, n_{2}^{2}\right)}, m_{2}\right)=1, \quad\left(\frac{m_{1}}{\left(m_{1}, n_{2}^{2}\right)}, \frac{n_{2}^{2}}{\left(m_{1}, n_{2}^{2}\right)}\right)=1
$$

we have

$$
\left(\frac{m_{1}}{\left(m_{1}, n_{2}^{2}\right)} n_{1}^{2}, m_{2} \frac{n_{2}^{2}}{\left(m_{1}, n_{2}^{2}\right)}\right)=\left(n_{1}^{2}, m_{2} \frac{n_{2}^{2}}{\left(m_{1}, n_{2}^{2}\right)}\right)=\left(n_{1}^{2}, m_{2}\right) .
$$

Thus, the conclusion of Lemma 15 follows.

Lemma 16. If $a$ is a positive integer, then

$$
\sum_{M<m \leq 2^{\varepsilon} M}(m, a) \ll M d(a) .
$$

Proof. We have

$$
\begin{aligned}
\sum_{M<m \leq 2^{\varepsilon} M}(m, a) & =\sum_{d \mid a} d \sum_{\substack{M<m \leq 2^{\varepsilon} M \\
(m, a)=d}} 1 \\
& =\sum_{d \mid a} d \sum_{\substack{M \\
\frac{M}{d}<m_{1} \leq \frac{2^{\varepsilon} M}{d} \\
\left(m_{1}, \frac{a}{d}\right)=1}} 1 \\
& \leq \sum_{d \mid a} d \sum_{\substack{M \\
d}} \sum_{m_{1} \leq \frac{2^{\varepsilon} M}{d}} 1 \\
& \ll \sum_{d \mid a} d \cdot \frac{M}{d}=M d(a) .
\end{aligned}
$$

Lemma 17. Suppose that $0<A<B<2 q$ and that $b$ is a positive integer. Then

$$
\sum_{\substack{A<a \leq B \\(a, q)=1 \\(a, b)=1}} e\left(l \frac{\bar{a}}{q}\right) \ll(l, q)^{\frac{1}{2}} q^{\frac{1}{2}+\varepsilon} b^{\varepsilon} .
$$

Here $\bar{a}$ is the integer such that $a \bar{a} \equiv 1(\bmod q)$.

Proof. By Lemma 3 of [4], for $0<A<B<2 q$, we have

$$
\sum_{\substack{A<a \leq B \\(a, q)=1}} e\left(l \frac{\bar{a}}{q}\right) \ll(l, q)^{\frac{1}{2}} q^{\frac{1}{2}+\varepsilon} .
$$


Hence,

$$
\begin{aligned}
\sum_{\substack{A<a \leq B \\
(a, q)=1 \\
(a, b)=1}} e\left(l \frac{\bar{a}}{q}\right)= & \sum_{\substack{A<a \leq B \\
(a, q)=1}}\left(\sum_{d \mid(a, b)} \mu(d)\right) e\left(l \frac{\bar{a}}{q}\right) \\
= & \sum_{d \mid b} \mu(d) \sum_{\substack{A<a \leq B \\
(a, q)=1 \\
d \mid a}} e\left(l \frac{\bar{a}}{q}\right) \\
= & \sum_{d \mid b} \mu(d) \sum_{\substack{\frac{A}{d}<t \leq \frac{B}{d} \\
(d t, q)=1}} e\left(l \cdot \frac{d \bar{t}}{q}\right) \\
= & \sum_{\substack{d \mid b \\
(d, q)=1}} \mu(d) \sum_{\substack{\frac{A}{d}<t \leq \frac{B}{d} \\
(t, q)=1}} e\left(l \bar{d} \cdot \frac{\bar{t}}{q}\right) \\
\ll & \sum_{\substack{d|b\\
|}} \mu(d) \mid \cdot(l \bar{d}, q)^{\frac{1}{2}} q^{\frac{1}{2}+\varepsilon} \\
& \ll(l, q)^{\frac{1}{2}} q^{\frac{1}{2}+\varepsilon} \sum_{d \mid b} 1 \\
\ll & (l, q)^{\frac{1}{2}} q^{\frac{1}{2}+\varepsilon} b^{\varepsilon} .
\end{aligned}
$$

Thus, Lemma 17 is proved.

\section{An asymptotic expression of $\zeta(1+i t)$}

Let

$$
\rho_{1}=\beta_{1}+i \gamma_{1}, \quad \rho_{2}=\beta_{2}+i \gamma_{2}, \quad \cdots, \quad \rho_{J}=\beta_{J}+i \gamma_{J}
$$

be all zeros of $\zeta(s)$ which satisfy $\beta \geq 1-4 \varepsilon, T \leq \gamma \leq 2 T$. By Lemma 12,

$$
J=N(1-4 \varepsilon, T, 2 T) \ll T^{11 \varepsilon} .
$$

We write domain $D$ as

$$
D=\{s=\sigma+i t: \quad 1-4 \varepsilon \leq \sigma, T \leq t \leq 2 T\}
$$


Write

$$
\begin{aligned}
& U_{1}=\bigcup_{j=1}^{J}\left(\gamma_{j}-(\log T)^{10}, \gamma_{j}+(\log T)^{10}\right), \\
& U_{2}=\bigcup_{j=1}^{J}\left(\gamma_{j}-2(\log T)^{10}, \gamma_{j}+2(\log T)^{10}\right), \\
& U_{3}=\bigcup_{j=1}^{J}\left(\gamma_{j}-3(\log T)^{10}, \gamma_{j}+3(\log T)^{10}\right), \\
& U_{4}=\bigcup_{j=1}^{J}\left(\gamma_{j}-4(\log T)^{10}, \gamma_{j}+4(\log T)^{10}\right) .
\end{aligned}
$$

After removing all domains of the form $\{s=\sigma+i t: 1-4 \varepsilon \leq \sigma<$ $\left.1, t \in U_{1}\right\}$ in $D$, we denote the remained domain as $D_{1}$. $D_{1}$ is a connected domain in which $\zeta(s) \neq 0$ so that we can define a holomorphic function $\log \zeta(s)$ in $D_{1}$. For $\operatorname{Re}(s)>1$, Euler's product formula produces

$$
\log \zeta(s)=-\sum_{p} \log \left(1-\frac{1}{p^{s}}\right)=\sum_{p} \sum_{m=1}^{\infty} \frac{1}{m p^{m s}}=\sum_{n=2}^{\infty} \frac{\Lambda_{1}(n)}{n^{s}},
$$

where

$$
\Lambda_{1}(n)=\frac{\Lambda(n)}{\log n} .
$$

After removing all domains of the form $\left\{s=\sigma+i t: 1-4 \varepsilon \leq \sigma, t \in U_{2}\right\}$ in $D$, we denote the remained domain as $D_{2}$. Now Lemma 1 can be applied. Take $f(z)=\log \zeta(z)$. For $s=\sigma+i t \in D_{2}, 1-2 \varepsilon \leq \sigma \leq 2$, let the center of circle be $z_{0}=2+i t$, the radius of bigger circle be $R=2-(1-4 \varepsilon)=1+4 \varepsilon$, the radius of smaller circle be $r=2-(1-2 \varepsilon)=1+2 \varepsilon$. On the bigger circle, by Lemma 7 ,

$$
\operatorname{Re} \log \zeta(z)=\log |\zeta(z)| \leq C \log T,
$$

where $C$ is a positive constant. Thus, for $s$ in the smaller circle, Lemma 1 yields

$$
|\log \zeta(s)| \leq \frac{2 r}{R-r} \cdot C \log T+\frac{R+r}{R-r} \cdot|\log \zeta(2+i t)| \ll \log T .
$$

For $\operatorname{Re}(s) \geq 2$, it is easy to see

$$
\log \zeta(s)=O(1)
$$


Hence, for $s=\sigma+i t \in D_{2}, \sigma \geq 1-2 \varepsilon$, we have

$$
|\log \zeta(s)| \ll \log T
$$

After removing all domains of the form $\left\{s=\sigma+i t: 1-4 \varepsilon \leq \sigma, t \in U_{3}\right\}$ in $D$, then limiting $\sigma \geq 1-2 \varepsilon$, we denote the obtained domain as $D_{3}$. Now Lemma 2 can be applied. Take $f(z)=\log \zeta(z)$. For $s=\sigma+i t \in D_{3}, 1-\varepsilon \leq$ $\sigma \leq 1+\varepsilon$, let the center of circle be $z_{0}=2+i t, R_{3}=2-(1-2 \varepsilon)=$ $1+2 \varepsilon, R_{2}=2-(1-\varepsilon)=1+\varepsilon, R_{1}=2-(1+\varepsilon)=1-\varepsilon$. By $(3.4)$, $M_{3} \ll \log T$. It is obvious that $M_{1}=O(1)$. Lemma 2 yields

$$
\begin{aligned}
\log M_{2} & \leq \frac{\log \left(\frac{1+2 \varepsilon}{1+\varepsilon}\right)}{\log \left(\frac{1+2 \varepsilon}{1-\varepsilon}\right)} \cdot \log M_{1}+\frac{\log \left(\frac{1+\varepsilon}{1-\varepsilon}\right)}{\log \left(\frac{1+2 \varepsilon}{1-\varepsilon}\right)} \cdot \log M_{3} \\
& \leq O(1)+\frac{2 \varepsilon+O\left(\varepsilon^{2}\right)}{3 \varepsilon+O\left(\varepsilon^{2}\right)} \cdot \log \log T \\
& =O(1)+\left(\frac{2}{3}+O(\varepsilon)\right) \log \log T \\
& \leq \frac{3}{4} \log \log T .
\end{aligned}
$$

Hence, for $s=\sigma+i t \in D_{3}, 1-\varepsilon \leq \sigma \leq 1+\varepsilon$, we have

$$
|\log \zeta(s)| \leq(\log T)^{\frac{3}{4}}
$$

For $\operatorname{Re}(s) \geq 1+\varepsilon$, it is obvious that

$$
\frac{1}{\zeta(s)}=O_{\varepsilon}(1)
$$

Thus, for $s=\sigma+i t \in D_{3}, \sigma \geq 1-\varepsilon$, we have

$$
\frac{1}{\zeta(s)} \ll \exp \left((\log T)^{\frac{3}{4}}\right) .
$$

After removing all domains of the form $\left\{s=\sigma+i t: 1-4 \varepsilon \leq \sigma, t \in U_{4}\right\}$ in $D$, then limiting $\sigma \geq 1-\varepsilon$, we denote the obtained domain as $D_{4}$. For $s \in D_{4}, u \geq 0,|v| \leq(\log T)^{3}$, we have

$$
\frac{1}{\zeta(s+u+i v)} \ll \exp \left((\log T)^{\frac{3}{4}}\right) .
$$


For $s=1+i t \in D_{4}, w=u+i v, X>1$, we have

$$
\begin{aligned}
& \frac{1}{2 \pi i} \int_{u=\varepsilon,|v| \leq(\log T)^{3}} \frac{1}{\zeta(s+w)} \cdot \Gamma(w) X^{w} d w \\
& =\frac{1}{2 \pi i} \int_{u=\varepsilon,|v| \leq(\log T)^{3}} \sum_{n=1}^{\infty} \frac{\mu(n)}{n^{s+w}} \cdot \Gamma(w) X^{w} d w \\
& =\sum_{n=1}^{\infty} \frac{\mu(n)}{n^{s}} \cdot \frac{1}{2 \pi i} \int_{u=\varepsilon,|v| \leq(\log T)^{3}} \Gamma(w)\left(\frac{X}{n}\right)^{w} d w .
\end{aligned}
$$

By Lemma 4, if $|v| \geq 1$, then on the vertical line $u=\epsilon$, we have

$$
\Gamma(w) \ll|v|^{\varepsilon-\frac{1}{2}} e^{-\frac{\pi}{2}|v|} .
$$

Hence,

$$
\begin{aligned}
& \frac{1}{2 \pi i} \int_{u=\varepsilon,|v|>(\log T)^{3}} \Gamma(w)\left(\frac{X}{n}\right)^{w} d w \\
& \ll\left(\frac{X}{n}\right)^{\varepsilon} \int_{u=\varepsilon,|v|>(\log T)^{3}}|\Gamma(w)||d w| \\
& \ll\left(\frac{X}{n}\right)^{\varepsilon} \int_{|v|>(\log T)^{3}}|v|^{\varepsilon-\frac{1}{2}} e^{-\frac{\pi}{2}|v|} d v \\
& \ll\left(\frac{X}{n}\right)^{\varepsilon} \int_{(\log T)^{3}}^{\infty} e^{-\frac{\pi}{2} v} d v \\
& \ll\left(\frac{X}{n}\right)^{\varepsilon} \exp \left(-\frac{\pi}{2}(\log T)^{3}\right) .
\end{aligned}
$$

By Lemma 3,

$$
\frac{1}{2 \pi i} \int_{\varepsilon-i \infty}^{\varepsilon+i \infty} \Gamma(w)\left(\frac{X}{n}\right)^{w} d w=e^{-\frac{n}{X}} .
$$

Therefore it follows that

$$
\begin{aligned}
& \frac{1}{2 \pi i} \int_{u=\varepsilon,|v| \leq(\log T)^{3}} \frac{1}{\zeta(s+w)} \cdot \Gamma(w) X^{w} d w \\
= & \sum_{n=1}^{\infty} \frac{\mu(n)}{n^{s}}\left(e^{-\frac{n}{X}}+O\left(\left(\frac{X}{n}\right)^{\varepsilon} \exp \left(-\frac{\pi}{2}(\log T)^{3}\right)\right)\right) \\
= & \sum_{n=1}^{\infty} \frac{\mu(n)}{n^{s}} e^{-\frac{n}{X}}+O\left(X^{\varepsilon} \exp \left(-\frac{\pi}{2}(\log T)^{3}\right)\right) .
\end{aligned}
$$

We move the line of integration to $\operatorname{Re}(w)=-\varepsilon$. At $w=0, \Gamma(w)$ has a pole of order 1 with residue 1 . Hence, the residue of $\frac{1}{\zeta(s+w)} \cdot \Gamma(w) X^{w}$ at 
$w=0$ is $\frac{1}{\zeta(s)}$. In two horizontal lines, by (3.6),

$$
\begin{aligned}
& \frac{1}{2 \pi i} \int_{-\varepsilon \leq u \leq \varepsilon,|v|=(\log T)^{3}} \frac{1}{\zeta(s+w)} \cdot \Gamma(w) X^{w} d w \\
& \ll X^{\varepsilon} \exp \left((\log T)^{\frac{3}{4}}\right) \int_{-\varepsilon}^{\varepsilon} e^{-\frac{\pi}{2}(\log T)^{3}} d u \\
& \ll X^{\varepsilon} \exp \left(-(\log T)^{3}\right) .
\end{aligned}
$$

The integration on $\operatorname{Re}(w)=-\varepsilon$ is

$$
\begin{aligned}
& \frac{1}{2 \pi i} \int_{u=-\varepsilon,|v| \leq(\log T)^{3}} \frac{1}{\zeta(s+w)} \cdot \Gamma(w) X^{w} d w \\
& \ll X^{-\varepsilon} \exp \left((\log T)^{\frac{3}{4}}\right)\left(\int_{u=-\varepsilon,|v| \leq(\log T)^{3}}|\Gamma(w)||d w|\right) \\
& \ll X^{-\varepsilon} \exp \left((\log T)^{\frac{3}{4}}\right)\left(\int_{u=-\varepsilon,|v| \leq 1}|\Gamma(w)||d w|\right. \\
& \left.+\int_{u=-\varepsilon, 1 \leq|v| \leq(\log T)^{3}}|\Gamma(w)||d w|\right) \\
& \ll X^{-\varepsilon} \exp \left((\log T)^{\frac{3}{4}}\right)\left(\int_{u=-\varepsilon,|v| \leq 1} \frac{|d w|}{|w|}\right. \\
& \left.+\int_{1 \leq|v| \leq(\log T)^{3}}|v|^{-\varepsilon-\frac{1}{2}} e^{-\frac{\pi}{2}|v|} d v\right) \\
& \ll \varepsilon X^{-\varepsilon} \exp \left((\log T)^{\frac{3}{4}}\right) .
\end{aligned}
$$

Combining all of the above, we get (with $s=1+i t$ )

$$
\begin{gathered}
\frac{1}{\zeta(s)}=\sum_{n=1}^{\infty} \frac{\mu(n)}{n^{s}} e^{-\frac{n}{X}}+O\left(X^{\varepsilon} \exp \left(-(\log T)^{3}\right)\right) \\
+O\left(X^{-\varepsilon} \exp \left((\log T)^{\frac{3}{4}}\right)\right) .
\end{gathered}
$$

Therefore we obtain an asymptotic expression of $\zeta(1+i t)$ as follows.

Proposition 1. Suppose that $T \leq t \leq 2 T, t \notin U_{4}$ and

$$
X=\exp \left(\frac{2}{\varepsilon}(\log T)^{\frac{3}{4}}\right)
$$

Then we have

$$
\frac{1}{\zeta(1+i t)}=\sum_{n \leq X} \frac{\mu(n)}{n^{1+i t}} e^{-\frac{n}{X}}+O(1)
$$

\section{A mean value estimate on $\zeta(\mathrm{s})$}


In this section, we shall prove the following mean value estimate on $\zeta(s)$.

Proposition 2. If $k$ is any given positive number, then we have

$$
\int_{1}^{T} \frac{\left|\zeta\left(\frac{1}{2}+i t\right)\right|^{4}}{|\zeta(1+2 i t)|^{k}} d t \ll_{k} T \log ^{4} T .
$$

Firstly we shall prove the following Proposition 3. We use the method of Iwaniec[4] essentially but with some modification and refinement.

Proposition 3. Suppose that $N \ll T^{\frac{1}{16}-\varepsilon}$ and that for $N<n \leq 2^{\varepsilon} N$, $a(n)=O\left(N^{-1+\varepsilon}\right)$. Then

$$
\int_{\frac{T}{2}}^{T}\left|\zeta\left(\frac{1}{2}+i t\right)\right|^{4}\left|\sum_{N<n \leq 2^{\varepsilon} N} \frac{a(n)}{n^{2 i t}}\right|^{2} d t \ll \frac{T \log ^{4} T}{N^{1-8 \varepsilon}} .
$$

Proof. By the discussion in Section 2 of [4], we shall estimate

$$
\begin{aligned}
& \log T \sum_{r \leq \frac{1}{2 \varepsilon \log 2} \log T+O(1)} \int_{0}^{\infty} e^{-\frac{t}{T}}\left|\zeta\left(\frac{1}{2}+i t\right)\right|^{2} . \\
& \cdot\left|\sum_{2^{\varepsilon r}<m \leq 2^{\varepsilon} \cdot 2^{\varepsilon r}} \frac{1}{m^{\frac{1}{2}+i t}}\right|^{2}\left|\sum_{N<n \leq 2^{\varepsilon} N} \frac{a(n)}{n^{2 i t}}\right|^{2} d t .
\end{aligned}
$$

Write

$$
\begin{aligned}
& \left|\left(\sum_{M<m \leq 2^{\varepsilon} M} \frac{1}{m^{\frac{1}{2}+i t}}\right)\left(\sum_{N<n \leq 2^{\varepsilon} N} \frac{a(n)}{n^{2 i t}}\right)\right|^{2} \\
& =\left|\sum_{K<k \leq 8^{\varepsilon} K} \frac{b(k)}{k^{i t}}\right|^{2}=\sum_{K<k, h \leq 8^{\varepsilon} K} b(k) \overline{b(h)}\left(\frac{h}{k}\right)^{i t},
\end{aligned}
$$

where $M=2^{\varepsilon r}, M \ll T^{\frac{1}{2}}, K=M N^{2}$,

$$
b(k)=\sum_{\substack{m n^{2}=k \\ M<m \leq 2^{\varepsilon} M \\ N<n \leq 2^{\varepsilon} N}} \frac{a(n)}{m^{\frac{1}{2}}} .
$$

In the following we shall estimate

$$
\begin{aligned}
& \int_{0}^{\infty} e^{-\frac{t}{T}}\left|\zeta\left(\frac{1}{2}+i t\right)\right|^{2}\left|\sum_{K<k \leq 8^{\varepsilon} K} \frac{b(k)}{k^{i t}}\right|^{2} \\
= & \sum_{K<k, h \leq 8^{\varepsilon} K} b(k) \overline{b(h)} \int_{0}^{\infty} e^{-\left(\frac{1}{T}-i \log \left(\frac{h}{k}\right)\right) t}\left|\zeta\left(\frac{1}{2}+i t\right)\right|^{2} d t .
\end{aligned}
$$


Let

$$
z=\frac{1}{T}-i \log \left(\frac{h}{k}\right)
$$

and note that

$$
|z| \leq \frac{1}{T}+\left|\log \left(\frac{h}{k}\right)\right|<4 \varepsilon
$$

for $K<k, h \leq 8^{\varepsilon} K$.

By Lemma 13,

$$
\begin{aligned}
& \int_{0}^{\infty} e^{-z t}\left|\zeta\left(\frac{1}{2}+i t\right)\right|^{2} d t \\
= & 2 \pi e^{\frac{i}{2 T}}\left(\frac{h}{k}\right)^{\frac{1}{2}} \sum_{l=1}^{\infty} d(l) \exp \left(2 \pi i l\left(\frac{h}{k}\right) e^{\frac{i}{T}}\right)+O(1) \\
= & 2 \pi e^{\frac{i}{2 T}}\left(\frac{h}{k}\right)^{\frac{1}{2}} \sum_{l=1}^{\infty} d(l) e\left(l \cdot \frac{h}{k}\right) \exp \left(2 \pi i l\left(\frac{h}{k}\right)\left(e^{\frac{i}{T}}-1\right)\right)+O(1) \\
= & 2 \pi e^{\frac{i}{2 T}}\left(\frac{h}{k}\right)^{\frac{1}{2}} \sum_{l=1}^{\infty} d(l) e\left(l \cdot \frac{h}{k}\right) \exp (2 \pi i l x)+O(1)
\end{aligned}
$$

where

$$
x=\frac{h}{k}\left(e^{\frac{i}{T}}-1\right) .
$$

The contribution of the term $O(1)$ to (4.1) is

$$
\begin{aligned}
& O\left(\sum_{K<k, h \leq 8^{\varepsilon} K}|b(k) b(h)|\right) \\
& \ll \sum_{M<m_{1} \leq 2^{\varepsilon} M} \frac{1}{m_{1}^{\frac{1}{2}}} \sum_{M<m_{2} \leq 2^{\varepsilon} M} \frac{1}{m_{2}^{\frac{1}{2}}} \sum_{N<n_{1} \leq 2^{\varepsilon} N}\left|a\left(n_{1}\right)\right| \sum_{N<n_{2} \leq 2^{\varepsilon} N}\left|a\left(n_{2}\right)\right| \\
& \ll M \sum_{N<n_{1} \leq 2^{\varepsilon} N} \frac{1}{N^{1-\varepsilon}} \sum_{N<n_{2} \leq 2^{\varepsilon} N} \frac{1}{N^{1-\varepsilon}} \\
& \ll M N^{2 \varepsilon} \ll \frac{T}{N^{1-8 \varepsilon}} .
\end{aligned}
$$

Let

$$
S\left(x ; \frac{h}{k}\right)=\sum_{l=1}^{\infty} d(l) e\left(l \cdot \frac{h}{k}\right) \exp (2 \pi i l x) .
$$

Write

$$
\mathfrak{z}=-2 \pi i x=4 \pi\left(\frac{h}{k}\right) \sin \left(\frac{1}{2 T}\right) e^{\frac{i}{2 T}} .
$$

By the discussion in Section 3 of [4], we know

$$
S\left(x ; \frac{h}{k}\right)=\frac{1}{2 \pi i} \int_{1+\varepsilon-i \infty}^{1+\varepsilon+i \infty} D\left(s ; \frac{h}{k}\right) \Gamma(s) \mathfrak{z}^{-s} d s,
$$


where

$$
D\left(s ; \frac{h}{k}\right)=\sum_{l=1}^{\infty} \frac{d(l)}{l^{s}} e\left(l \frac{h}{k}\right) .
$$

In the following we write

$$
k^{*}=\frac{k}{(k, h)}, \quad h^{*}=\frac{h}{(k, h)} .
$$

We move the line of integration from $\operatorname{Re}(s)=1+\varepsilon$ to $\operatorname{Re}(s)=-\varepsilon$, and get

$$
\begin{aligned}
S\left(x ; \frac{h}{k}\right) & =\frac{1}{2 \pi i} \int_{1+\varepsilon-i \infty}^{1+\varepsilon+i \infty} D\left(s ; \frac{h^{*}}{k^{*}}\right) \Gamma(s) \mathfrak{z}^{-s} d s \\
& =\frac{1}{2 \pi i} \int_{-\varepsilon-i \infty}^{-\varepsilon+i \infty} D\left(s ; \frac{h^{*}}{k^{*}}\right) \Gamma(s) \mathfrak{z}^{-s} d s+R_{1}(T ; h, k)+R_{0}(T ; h, k) \\
& =R(T ; h, k)+R_{1}(T ; h, k)+R_{0}(T ; h, k),
\end{aligned}
$$

where

$$
R(T ; h, k)=\frac{1}{2 \pi i} \int_{-\varepsilon-i \infty}^{-\varepsilon+i \infty} D\left(s ; \frac{h^{*}}{k^{*}}\right) \Gamma(s) \mathfrak{z}^{-s} d s,
$$

$R_{1}(T ; h, k)$ and $R_{0}(T ; h, k)$ are residues of $D\left(s ; \frac{h^{*}}{k^{*}}\right) \Gamma(s) \mathfrak{z}^{-s}$ coming from the poles at $s=1$ and $s=0$ respectively.

By the discussion in Section 3 of [4] and Lemma 14, we know that

$$
\begin{aligned}
R_{1}(T ; h, k) & =\frac{1}{\mathfrak{z} k^{*}}\left(\gamma-\log \mathfrak{z}-2 \log k^{*}\right) \ll \frac{T \log T}{k^{*}}, \\
R_{0}(T ; h, k) & =D\left(0 ; \frac{h^{*}}{k^{*}}\right) \\
& =\frac{1}{4}-\frac{1}{\pi i} \sum_{a=1}^{k^{*}} \beta\left(a, k^{*}\right) \sum_{0<b<\frac{k^{*}}{2}} \eta\left(b, k^{*}\right) e\left(a b \frac{\overline{h^{*}}}{k^{*}}\right) .
\end{aligned}
$$

Now we see the contribution of $R_{1}(T ; h, k), R(T ; h, k)$ and $R_{0}(T ; h, k)$ to (4.1).

1. The contribution of $R_{1}(T ; h, k)$

We note that $\frac{h}{k} \ll 1$ for $K<h, k \leq 8^{\varepsilon} K$. Therefore the contribution of $R_{1}(T ; h, k)$ is

$$
\begin{aligned}
& \ll \sum_{K<k, h \leq 8^{\varepsilon} K}|b(k) b(h)|\left|R_{1}(T ; h, k)\right| \\
& \ll \sum_{K<k, h \leq 8^{\varepsilon} K}|b(k) b(h)| \cdot \frac{T \log T}{k}(k, h)
\end{aligned}
$$




$$
\begin{aligned}
& \ll T \log T \sum_{M<m_{1} \leq 2^{\varepsilon}} \sum_{M} \sum_{M<m_{2} \leq 2^{\varepsilon}} \sum_{M<n_{1} \leq 2^{\varepsilon} N} \sum_{N<n_{2} \leq 2^{\varepsilon} N} \frac{\left|a\left(n_{1}\right)\right|}{m_{1}^{\frac{1}{2}}} . \\
& \cdot \frac{\left|a\left(n_{2}\right)\right|}{m_{2}^{\frac{1}{2}}} \cdot \frac{1}{m_{1} n_{1}^{2}}\left(m_{1} n_{1}^{2}, m_{2} n_{2}^{2}\right) \\
& \ll T \log T \cdot \frac{1}{M N^{2-2 \varepsilon}} \cdot \frac{1}{M N^{2}} \sum_{M<m_{1} \leq 2^{\varepsilon}} \sum_{M} \cdot \\
& \cdot \sum_{N<n_{1} \leq 2^{\varepsilon} N} \sum_{N<n_{2} \leq 2^{\varepsilon} N}\left(m_{1} n_{1}^{2}, m_{2} n_{2}^{2}\right) \\
& =\frac{T \log T}{M^{2} N^{4-2 \varepsilon}} \sum_{M<m_{1} \leq 2^{\varepsilon}} \sum_{M} \sum_{M<m_{2} \leq 2^{\varepsilon}} \sum_{M} \sum_{N<n_{1} \leq 2^{\varepsilon} N}\left(m_{1} n_{1}^{2}, m_{2} n_{2}^{2}\right) .
\end{aligned}
$$

By Lemmas 15 and 16,

$$
\begin{aligned}
& \sum_{M<m_{1} \leq 2^{\varepsilon}} \sum_{M} \sum_{M<m_{2} \leq 2^{\varepsilon}} \sum_{M} \sum_{N<n_{1} \leq 2^{\varepsilon} N}\left(m_{1} n_{1}^{2}, m_{2} n_{2}^{2}\right) \\
& =\sum_{d \leq 2^{\varepsilon}} \sum_{M} \sum_{M<m_{1} \leq 2^{\varepsilon}} \sum_{M} \sum_{\begin{array}{c}
M<m_{2} \leq 2^{\varepsilon} M \\
\left(m_{1}, m_{2}\right)=d
\end{array}} \sum_{r \leq 2^{\varepsilon} N} . \\
& \sum_{\substack{N<n_{2} \leq 2^{\varepsilon} N \\
\left(n_{1}, n_{2}\right)=r}}\left(m_{1} n_{1}^{2}, m_{2} n_{2}^{2}\right) \\
& =\sum_{d \leq 2^{\varepsilon} M} d \sum_{\frac{M}{d}<m_{1}^{\prime} \leq \frac{2^{\varepsilon} M}{d}} \sum_{\substack{\frac{M}{d}<m_{2}^{\prime} \leq \frac{2^{\varepsilon} M}{d} \\
\left(m_{1}^{\prime}, m_{2}^{\prime}\right)=1}} \sum_{r \leq 2^{\varepsilon} N} r^{2} \sum_{\frac{N}{r}<n_{1}^{\prime} \leq \frac{2^{\varepsilon} N}{r}} . \\
& \cdot \sum_{\substack{N \\
\frac{N}{r}<n_{2}^{\prime} \leq \frac{2^{\varepsilon} N}{r} \\
\left(n_{1}^{\prime}, n_{2}^{\prime}\right)=1}}\left(m_{1}^{\prime} n_{1}^{\prime 2}, m_{2}^{\prime} n_{2}^{\prime 2}\right) \\
& =\sum_{d \leq 2^{\varepsilon} M} d \sum_{\frac{M}{d}<m_{1}^{\prime} \leq \frac{2^{\varepsilon} M}{d}} \sum_{\substack{\frac{M}{d}<m_{2}^{\prime} \leq \frac{2^{\varepsilon} M}{d} \\
\left(m_{1}^{\prime}, m_{2}^{\prime}\right)=1}} \sum_{r \leq 2^{\varepsilon} N} r^{2} \sum_{\substack{\frac{N}{r}<n_{1}^{\prime} \leq \frac{2^{\varepsilon} N}{r} \\
.}} . \\
& \cdot \sum_{\substack{N \\
\frac{N}{r}<n_{2}^{\prime} \leq \frac{2^{\varepsilon} N}{r} \\
\left(n_{1}^{\prime}, n_{2}^{\prime}\right)=1}}\left(m_{1}^{\prime}, n_{2}^{\prime 2}\right)\left(m_{2}^{\prime}, n_{1}^{\prime 2}\right) \\
& \leq \sum_{d \leq 2^{\varepsilon} M} d \sum_{\frac{M}{d}<m_{1}^{\prime} \leq \frac{2^{\varepsilon} M}{d}} \sum_{\frac{M}{d}<m_{2}^{\prime} \leq \frac{2^{\varepsilon} M}{d}} \sum_{r \leq 2^{\varepsilon} N} r^{2} \sum_{\frac{N}{r}<n_{1}^{\prime} \leq \frac{2^{\varepsilon} N}{r}} . \\
& \sum_{\frac{N}{r}<n_{2}^{\prime} \leq \frac{2^{\varepsilon} N}{r}}\left(m_{1}^{\prime}, n_{2}^{\prime 2}\right)\left(m_{2}^{\prime}, n_{1}^{\prime 2}\right)
\end{aligned}
$$




$$
\begin{aligned}
& =\sum_{d \leq 2^{\varepsilon} M} d \sum_{r \leq 2^{\varepsilon} N} r^{2} \sum_{\frac{N}{r}<n_{2}^{\prime} \leq 2^{\varepsilon} N} \sum_{\frac{M}{d}<m_{1}^{\prime} \leq \frac{2^{\varepsilon} M}{d}}\left(m_{1}^{\prime}, n_{2}^{\prime 2}\right) . \\
& \qquad \sum_{\frac{N}{r}<n_{1}^{\prime} \leq 2^{\varepsilon} N} \sum_{\frac{M}{d}<m_{2}^{\prime} \leq \frac{2^{\varepsilon} M}{d}}\left(m_{2}^{\prime}, n_{1}^{\prime 2}\right) \\
& \ll \sum_{d \leq 2^{\varepsilon} M} d \sum_{r \leq 2^{\varepsilon} N} r^{2} \sum_{\frac{N}{r}<n_{2}^{\prime} \leq \frac{2^{\varepsilon} N}{r}} \frac{M}{d} \cdot d\left(n_{2}^{\prime 2}\right) \sum_{\frac{N}{r}<n_{1}^{\prime} \leq \frac{2^{\varepsilon} N}{r}} \frac{M}{d} \cdot d\left(n_{1}^{\prime 2}\right) \\
& \ll M^{2} N^{2 \varepsilon} \sum_{d \leq 2^{\varepsilon} M} \frac{1}{d} \sum_{r \leq 2^{\varepsilon} N} r^{2} \sum_{\frac{N}{r}<n_{1}^{\prime} \leq \frac{2^{\varepsilon} N}{r}} \sum_{\frac{N}{r}<n_{2}^{\prime} \leq \frac{2^{\varepsilon} N}{r}} 1 \\
& \ll M^{2} N^{2 \varepsilon} \log (2 M) \sum_{r \leq 2^{\varepsilon} N} r^{2}\left(\frac{N}{r}\right)^{2} \\
& \ll M^{2} N^{3+2 \varepsilon} \log (2 M) .
\end{aligned}
$$

Hence, the contribution of $R_{1}(T ; h, k)$ is

$$
\ll \frac{T \log T}{M^{2} N^{4-2 \varepsilon}} \cdot M^{2} N^{3+2 \varepsilon} \log (2 M) \ll \frac{T \log ^{2} T}{N^{1-8 \varepsilon}} .
$$

2. The contribution of $R(T ; h, k)$

By the functional equation in Lemma 14, we get

$$
\begin{aligned}
R(T ; h, k)= & \frac{1}{2 \pi i} \int_{-\varepsilon-i \infty}^{-\varepsilon+i \infty} D\left(s ; \frac{h^{*}}{k^{*}}\right) \Gamma(s) \mathfrak{z}^{-s} d s \\
= & \frac{1}{2 \pi i} \int_{-\varepsilon-i \infty}^{-\varepsilon+i \infty} 2 G^{2}(s) k^{*(1-2 s)}\left(D\left(1-s ; \frac{\overline{h^{*}}}{k^{*}}\right)\right. \\
& \left.-\cos (\pi s) D\left(1-s ;-\frac{h^{*}}{k^{*}}\right)\right) \Gamma(s) \mathfrak{z}^{-s} d s \\
= & k^{*} \sum_{l=1}^{\infty} \frac{d(l)}{l} \cdot \frac{1}{2 \pi i} \int_{-\varepsilon-i \infty}^{-\varepsilon+i \infty} 2 G^{2}(s) \cdot \frac{l^{s}}{\left(h^{*} k^{*}\right)^{s}} \cdot \\
& \cdot\left(e\left(l \frac{\overline{h^{*}}}{k^{*}}\right)-\cos (\pi s) e\left(-l \frac{\overline{h^{*}}}{k^{*}}\right)\right) \Gamma(s)\left(4 \pi \sin \left(\frac{1}{2 T}\right) e^{\frac{i}{2 T}}\right)^{-s} d s \\
= & k^{*} \sum_{l=1}^{\infty} \frac{d(l)}{l} \cdot \frac{1}{2 \pi i} \int_{-\varepsilon-i \infty}^{-\varepsilon+i \infty} U(s, T)\left(\frac{l}{h^{*} k^{*}}\right)^{s} . \\
& \cdot\left(e\left(l \frac{h^{*}}{k^{*}}\right)-\cos (\pi s) e\left(-l \frac{h^{*}}{k^{*}}\right)\right) d s,
\end{aligned}
$$

where

$$
U(s, T)=2 G^{2}(s) \Gamma(s)\left(4 \pi \sin \left(\frac{1}{2 T}\right) e^{\frac{i}{2 T}}\right)^{-s} .
$$

The contribution of $R(T ; h, k)$ is

$$
\ll\left|\sum_{K<k, h \leq 8^{\varepsilon} K} b(k) \overline{b(h)}\left(\frac{h}{k}\right)^{\frac{1}{2}} R(T ; h, k)\right|,
$$


while

$$
\begin{aligned}
& \sum_{K<k, h \leq 8^{\varepsilon} K} b(k) \overline{b(h)}\left(\frac{h}{k}\right)^{\frac{1}{2}} R(T ; h, k) \\
= & \sum_{K<k \leq 8^{\varepsilon} K} \sum_{K<h \leq 8^{\varepsilon} K} \frac{b(k)}{k^{\frac{1}{2}}} \cdot \overline{b(h)} h^{\frac{1}{2}} \cdot k^{*} \sum_{l=1}^{\infty} \frac{d(l)}{l} . \\
& \cdot \frac{1}{2 \pi i} \int_{-\varepsilon-i \infty}^{-\varepsilon+i \infty} U(s, T)\left(\frac{l}{h^{*} k^{*}}\right)^{s}\left(e\left(l \frac{\overline{h^{*}}}{k^{*}}\right)-\cos (\pi s) e\left(-l \frac{h^{*}}{k^{*}}\right)\right) d s \\
= & \sum_{l=1}^{\infty} \frac{d(l)}{l} \cdot \frac{1}{2 \pi i} \int_{-\varepsilon-i \infty}^{-\varepsilon+i \infty} U(s, T) l^{s}\left(\sum_{K<k \leq 8^{\varepsilon} K} \sum_{K<h \leq 8^{\varepsilon} K} \frac{b(k)}{k^{\frac{1}{2}}} \cdot \overline{b(h)} h^{\frac{1}{2}} .\right. \\
& \cdot \frac{k^{*}}{\left(h^{*} k^{*}\right)^{s}} e\left(l \frac{k^{*}}{k^{*}}\right)-\cos (\pi s) \quad \sum_{K<k \leq 8^{\varepsilon} K K<h \leq 8^{\varepsilon} K} \frac{b(k)}{k^{\frac{1}{2}}} \cdot \overline{b(h)} h^{\frac{1}{2}} . \\
= & \sum_{l=1}^{\infty} \frac{d(l)}{l} \cdot \frac{1}{2 \pi i} \int_{-\varepsilon-i \infty}^{-\varepsilon+i \infty} U(s, T) l^{s}(Q(l, s)-\cos (\pi s) Q(-l, s)) d s,
\end{aligned}
$$

where

$$
\begin{aligned}
Q(l, s) & =\sum_{K<k \leq 8^{\varepsilon} K} \sum_{K<h \leq 8^{\varepsilon} K} \frac{b(k)}{k^{\frac{1}{2}}} \cdot \overline{b(h)} h^{\frac{1}{2}} \cdot \frac{k^{*}}{\left(h^{*} k^{*}\right)^{s}} e\left(l \frac{\overline{h^{*}}}{k^{*}}\right) \\
& =\sum_{K<k \leq 8^{\varepsilon} K} \sum_{K<h \leq 8^{\varepsilon} K} b(k) \overline{b(h)} \cdot \frac{1}{\left(k^{*} h^{*}\right)^{s-\frac{1}{2}}} e\left(l \frac{h^{*}}{k^{*}}\right) .
\end{aligned}
$$

For $s=-\varepsilon+i t$, by the discussion in Section 5 of [4],

$$
\begin{aligned}
& U(s, T) l^{s} \ll\left(\frac{T}{l}\right)^{\varepsilon}(|t|+1)^{\frac{1}{2}+\varepsilon} \exp \left(\left(\frac{1}{2 T}-\frac{3}{2} \pi\right)|t|\right), \\
& U(s, T) l^{s} \cos (\pi s) \ll\left(\frac{T}{l}\right)^{\varepsilon}(|t|+1)^{\frac{1}{2}+\varepsilon} \exp \left(\left(\frac{1}{2 T}-\frac{\pi}{2}\right)|t|\right) .
\end{aligned}
$$

In the following we shall estimate $Q(l, s)$ for $s=-\varepsilon+i t$.

$$
\begin{aligned}
Q(l, s) & =\sum_{K<k \leq 8^{\varepsilon} K} \sum_{K<h \leq 8^{\varepsilon} K} b(k) \overline{b(h)} \cdot \frac{1}{\left(k^{*} h^{*}\right)^{s-\frac{1}{2}}} e\left(l \frac{\overline{h^{*}}}{k^{*}}\right) \\
& =\sum_{K<k \leq 8^{\varepsilon} K} \sum_{K<h \leq 8^{\varepsilon} K} b(k) \overline{b(h)} \cdot \frac{(k, h)^{2 s-1}}{(k h)^{s-\frac{1}{2}}} e\left(l \frac{\overline{h^{*}}}{k^{*}}\right) \\
& =\sum_{d \leq 8^{\varepsilon} K} d^{2 s-1} \sum_{K<k \leq 8^{\varepsilon} K} \sum_{\substack{K<h \leq 8^{\varepsilon} K \\
(k, h)=d}} \frac{b(k) \overline{b(h)}}{(k h)^{s-\frac{1}{2}}} e\left(l \frac{\left(\frac{h}{d}\right)}{\frac{k}{d}}\right)
\end{aligned}
$$




$$
\begin{aligned}
& =\sum_{d \leq 8^{\varepsilon} K} d^{2 s-1} \sum_{N<n_{1} \leq 2^{\varepsilon} N} \sum_{N<n_{2} \leq 2^{\varepsilon}} \sum_{N<m_{1} \leq 2^{\varepsilon} M} . \\
& \sum_{\substack{M<m_{2} \leq 2^{\varepsilon} M \\
\left(m_{1} n_{1}^{2}, m_{2} n_{2}^{2}\right)=d}} \frac{a\left(n_{2}\right)}{m_{2}^{\frac{1}{2}}} \cdot \frac{\overline{a\left(n_{1}\right)}}{m_{1}^{\frac{1}{2}}} \cdot \frac{1}{\left(m_{1} m_{2} n_{1}^{2} n_{2}^{2}\right)^{s-\frac{1}{2}}} e\left(l \frac{\overline{\left(\frac{m_{1} n_{1}^{2}}{d}\right)}}{\frac{m_{2} n_{2}^{2}}{d}}\right) \\
& =\sum_{d \leq 8^{\varepsilon} K} d^{2 s-1} \sum_{N<n_{1} \leq 2^{\varepsilon} N} \sum_{N<n_{2} \leq 2^{\varepsilon} N} \frac{a\left(n_{2}\right) \overline{a\left(n_{1}\right)}}{\left(n_{1} n_{2}\right)^{2 s-1}} . \\
& \cdot \sum_{M<m_{1} \leq 2^{\varepsilon} M} \sum_{\substack{M<m_{2} \leq 2^{\varepsilon} M \\
\left(m_{1} n_{1}^{2}, m_{2} n_{2}^{2}\right)=d}} \frac{1}{\left(m_{1} m_{2}\right)^{s}} e\left(l \overline{\frac{\left(\frac{m_{1} n_{1}^{2}}{d}\right)}{m_{2} n_{2}^{2}}}\right) \\
& =\sum_{d \leq 8^{\varepsilon} K} d^{2 s-1} \sum_{N<n_{1} \leq 2^{\varepsilon} N} \sum_{N<n_{2} \leq 2^{\varepsilon} N} \frac{a\left(n_{2}\right) \overline{a\left(n_{1}\right)}}{\left(n_{1} n_{2}\right)^{2 s-1}} \cdot B\left(l, s, n_{1}, n_{2}, d\right),
\end{aligned}
$$

where

$$
B\left(l, s, n_{1}, n_{2}, d\right)=\sum_{\substack{M<m_{2} \leq 2^{\varepsilon} M \\ d \mid m_{2} n_{2}^{2}}} \sum_{\substack{M<m_{1} \leq 2^{\varepsilon} M \\\left(m_{1} n_{1}^{2}, m_{2} n_{2}^{2}\right)=d}} \frac{1}{\left(m_{1} m_{2}\right)^{s}} e\left(l \overline{\frac{\left(\frac{m_{1} n_{1}^{2}}{d}\right)}{\frac{m_{2} n_{2}^{2}}{d}}}\right) .
$$

We shall estimate

$$
\sum_{\substack{M<m_{1} \leq M_{1} \\\left(m_{1} n_{1}^{2}, m_{2} n_{2}^{2}\right)=d}} e\left(l \overline{\left.\frac{\left(\frac{m_{1} n_{1}^{2}}{d}\right)}{\frac{m_{2} n_{2}^{2}}{d}}\right)}\right.
$$

for $M<M_{1} \leq 2^{\varepsilon} M$. Let $\left(m_{1}, d\right)=d_{1}$. Write $d=d_{1} d_{2}$. We see $\left(d_{2}, \frac{m_{1}}{d_{1}}\right)=$ 1. Hence, $d\left|m_{1} n_{1}^{2} \Longrightarrow d_{2}\right| n_{1}^{2} \Longrightarrow d_{2} \leq 4^{\varepsilon} N^{2}$. By Lemma 17 ,

$$
\begin{aligned}
& \sum_{\substack{\left.M<m_{1} \leq M_{1} \\
m_{1} n_{1}^{2}, m_{2} n_{2}^{2}\right)=d}} e\left(\overline{\left.\frac{\left(\frac{m_{1} n_{1}^{2}}{d}\right)}{\frac{m_{2} n_{2}^{2}}{d}}\right)}\right. \\
& =\sum_{d_{1} \mid d}\left(\sum_{M<m_{1} \leq M_{1}} e\left(l \overline{\frac{\left(\frac{m_{1} n_{1}^{2}}{d}\right)}{m_{2} n_{2}^{2}}}\right)\right) \\
& \begin{array}{c}
\left(m_{1}, d\right)=d_{1} \\
\left(\frac{m_{1}}{d_{1}} \cdot \frac{n_{1}^{2}}{d_{2}}, \frac{m_{2} n_{2}^{2}}{d}\right)=1
\end{array}
\end{aligned}
$$

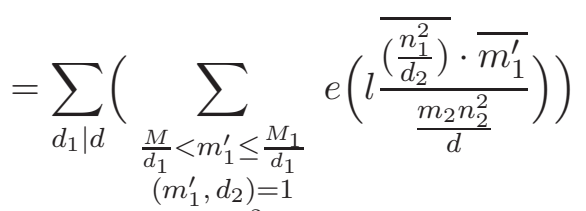

$$
\begin{aligned}
& \left(m_{1}^{\prime}, \frac{m_{2} n_{2}^{2}}{d}\right)=1 \\
& \left(\frac{n_{1}^{2}}{d_{2}}, \frac{m_{2} n_{2}^{2}}{d}\right)=1
\end{aligned}
$$




$$
\begin{aligned}
= & \sum_{\substack{d_{1} \mid d \\
\left(\frac{n_{1}^{2}}{d_{2}}, \frac{m_{2} n_{2}^{2}}{d}\right)=1}}\left(\sum_{\substack{\frac{M}{d_{1}}<m_{1}^{\prime} \leq \frac{M_{1}}{d_{1}} \\
\left(m_{1}^{\prime}, \frac{m_{2} n_{2}^{2}}{d}\right)=1 \\
\left(m_{1}^{\prime}, d_{2}\right)=1}} e\left(l\left(\overline{\frac{n_{1}^{2}}{d_{2}}}\right) \cdot \frac{\overline{m_{1}^{\prime}}}{\frac{m_{2} n_{2}^{2}}{d}}\right)\right) \\
\ll & \sum_{\left(\frac{n_{1}^{2}}{d_{2}}, \frac{m_{2} n_{2}^{2}}{d}\right)=1}\left(l\left(\frac{n_{1}^{2}}{d_{2}}\right), \frac{m_{2} n_{2}^{2}}{d}\right)^{\frac{1}{2}}\left(\frac{m_{2} n_{2}^{2}}{d}\right)^{\frac{1}{2}+\varepsilon} d_{2}^{\varepsilon} \\
\ll & \left(l, \frac{m_{2} n_{2}^{2}}{d}\right)^{\frac{1}{2}} \sum_{d_{1} \mid d}\left(\frac{m_{2} n_{2}^{2}}{d}\right)^{\frac{1}{2}+\varepsilon} d_{2}^{\varepsilon} \\
\ll & \left(\sum_{d_{1} \mid d} 1\right)\left(l, \frac{m_{2} n_{2}^{2}}{d}\right)^{\frac{1}{2}}\left(\frac{M N^{2}}{d}\right)^{\frac{1}{2}+\varepsilon} d^{\varepsilon} \\
\ll & \left(l, \frac{m_{2} n_{2}^{2}}{d}\right)^{\frac{1}{2}}\left(\frac{M N^{2}}{d}\right)^{\frac{1}{2}+\varepsilon} d^{2 \varepsilon},
\end{aligned}
$$

here we note $d_{2} \leq 4^{\varepsilon} N^{2} \Longrightarrow \frac{M_{1}}{d_{1}}<\frac{2 m_{2} n_{2}^{2}}{d}$.

By the above estimate and partial summation, for $s=-\varepsilon+i t$, we have

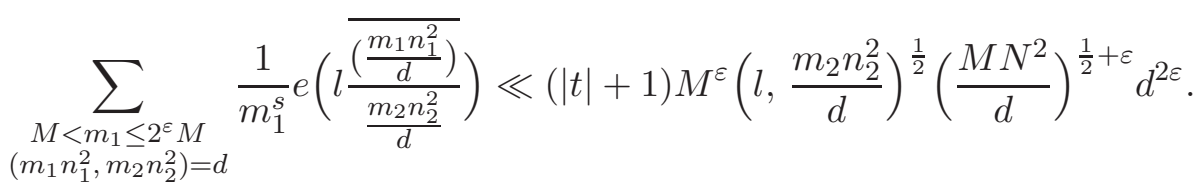

By Lemma 16,

$$
\begin{aligned}
B\left(l, s, n_{1}, n_{2}, d\right) & \ll(|t|+1)\left(\frac{M N^{2}}{d}\right)^{\frac{1}{2}+\varepsilon} M^{2 \varepsilon} d^{2 \varepsilon} \sum_{\substack{M<m_{2} \leq 2^{\varepsilon} M \\
d \mid m_{2} n_{2}^{2}}}\left(l, \frac{m_{2} n_{2}^{2}}{d}\right)^{\frac{1}{2}} \\
& \leq(|t|+1)\left(\frac{M N^{2}}{d}\right)^{\frac{1}{2}+\varepsilon} M^{2 \varepsilon} d^{2 \varepsilon} \sum_{\substack{M<m_{2} \leq 2^{\varepsilon} M\\
}}\left(l, m_{2}\right)^{\frac{1}{2}}\left(l, n_{2}^{2}\right)^{\frac{1}{2}} \\
& \ll(|t|+1)\left(\frac{M N^{2}}{d}\right)^{\frac{1}{2}+\varepsilon} M^{1+2 \varepsilon} d^{2 \varepsilon}\left(l, n_{2}^{2}\right)^{\frac{1}{2}} l^{\frac{\varepsilon}{4}} .
\end{aligned}
$$

By Lemma 16 again, we get

$$
\begin{aligned}
Q(l, s) & \ll(|t|+1)\left(M N^{2}\right)^{\frac{1}{2}+\varepsilon} M^{1+2 \varepsilon} \sum_{d \leq 8^{\varepsilon} K} \frac{1}{d^{\frac{3}{2}+\varepsilon}} . \\
& \cdot \sum_{N<n_{1} \leq 2^{\varepsilon} N} \sum_{N<n_{2} \leq 2^{\varepsilon} N}\left|a\left(n_{1}\right) a\left(n_{2}\right)\right| N^{2(1+2 \varepsilon)}\left(l, n_{2}^{2}\right)^{\frac{1}{2} l^{\frac{\varepsilon}{4}}} \\
& \ll(|t|+1) M^{\frac{3}{2}+3 \varepsilon} N^{2+8 \varepsilon} \sum_{N<n_{2} \leq 2^{\varepsilon} N}\left(n_{2}, l\right) l^{\frac{\varepsilon}{4}} \\
& \ll(|t|+1) M^{\frac{3}{2}+3 \varepsilon} N^{3+8 \varepsilon} l^{\frac{\varepsilon}{2}} .
\end{aligned}
$$


The contribution of $R(T ; h, k)$ is

$$
\begin{aligned}
& \ll \sum_{l=1}^{\infty} \frac{d(l)}{l}\left(\frac{T}{l}\right)^{\varepsilon} \int_{-\infty}^{\infty}(|t|+1)^{\frac{3}{2}+\varepsilon} \exp \left(\left(\frac{1}{2 T}-\frac{\pi}{2}\right)|t|\right) d t \cdot M^{\frac{3}{2}+3 \varepsilon} N^{3+8 \varepsilon} l^{\frac{\varepsilon}{2}} \\
& \ll T^{\varepsilon} M^{\frac{3}{2}+3 \varepsilon} N^{3+8 \varepsilon} \sum_{l=1}^{\infty} \frac{d(l)}{l^{1+\frac{\varepsilon}{2}}} \\
& \ll T^{\varepsilon} M^{\frac{3}{2}+3 \varepsilon} N^{3+8 \varepsilon} \\
& \ll \frac{T}{N^{1-8 \varepsilon}} .
\end{aligned}
$$

3. The contribution of $R_{0}(T ; h, k)$

Using Lemma 14, (4.12) and the estimates in 2., we get that the contribution of $R_{0}(T ; h, k)$ is

$$
\ll\left|\sum_{K<k, h \leq 8^{\varepsilon} K} b(k) \overline{b(h)}\left(\frac{h}{k}\right)^{\frac{1}{2}} R_{0}(T ; h, k)\right|,
$$

while

$$
\begin{aligned}
& \sum_{K<k, h \leq 8^{\varepsilon} K} b(k) \overline{b(h)}\left(\frac{h}{k}\right)^{\frac{1}{2}} R_{0}(T ; h, k) \\
& =\sum_{K<k \leq 8^{\varepsilon} K} \sum_{K<h \leq 8^{\varepsilon} K} b(k) \overline{b(h)}\left(\frac{h}{k}\right)^{\frac{1}{2}}\left(\frac{1}{4}-\frac{1}{\pi i} \sum_{a=1}^{k^{*}} \beta\left(a, k^{*}\right) .\right. \\
& \left.\cdot \sum_{0<b<\frac{k^{*}}{2}} \eta\left(b, k^{*}\right) e\left(a b \frac{\overline{h^{*}}}{k^{*}}\right)\right) \\
& =\sum_{d \leq 8^{\varepsilon} K} \sum_{K<k \leq 8^{\varepsilon} K} \sum_{\substack{K<h \leq 8^{\varepsilon} K \\
(k, h)=d}} b(k) \overline{b(h)}\left(\frac{h}{k}\right)^{\frac{1}{2}}\left(\frac{1}{4}-\frac{1}{\pi i} \sum_{a=1}^{\frac{k}{d}} \beta\left(a, \frac{k}{d}\right) .\right. \\
& \left.\sum_{0<b<\frac{k}{2 d}} \eta\left(b, \frac{k}{d}\right) e\left(a b \frac{\overline{\left(\frac{h}{d}\right)}}{\frac{k}{d}}\right)\right) \\
& =\sum_{d \leq 8^{\varepsilon} K} \sum_{N<n_{1} \leq 2^{\varepsilon}} \sum_{N} \sum_{N<n_{2} \leq 2^{\varepsilon} N} \sum_{M<m_{2} \leq 2^{\varepsilon} M} \sum_{\substack{M<m_{1} \leq 2^{\varepsilon} M \\
\left(m_{1} n_{1}^{2}, m_{2} n_{2}^{2}\right)=d}} \frac{a\left(n_{2}\right)}{m_{2}^{\frac{1}{2}}} \cdot \frac{\overline{a\left(n_{1}\right)}}{m_{1}^{\frac{1}{2}}} . \\
& \cdot\left(\frac{m_{1} n_{1}^{2}}{m_{2} n_{2}^{2}}\right)^{\frac{1}{2}}\left(\frac{1}{4}-\frac{1}{\pi i} \sum_{a=1}^{\frac{m_{2} n_{2}^{2}}{d}} \beta\left(a, \frac{m_{2} n_{2}^{2}}{d}\right) \sum_{0<b<\frac{m_{2} n_{2}^{2}}{2 d}} \eta\left(b, \frac{m_{2} n_{2}^{2}}{d}\right) e\left(a b \frac{\overline{\left(\frac{m_{1} n_{1}^{2}}{d}\right)}}{\frac{m_{2} n_{2}^{2}}{d}}\right)\right) \\
& =\sum_{d \leq 8^{\varepsilon} K} \sum_{N<n_{1} \leq 2^{\varepsilon} N} \sum_{N<n_{2} \leq 2^{\varepsilon} N} a\left(n_{2}\right) \overline{a\left(n_{1}\right)}\left(\frac{n_{1}}{n_{2}}\right) \sum_{M<m_{2} \leq 2^{\varepsilon} M} \frac{1}{m_{2}} .
\end{aligned}
$$




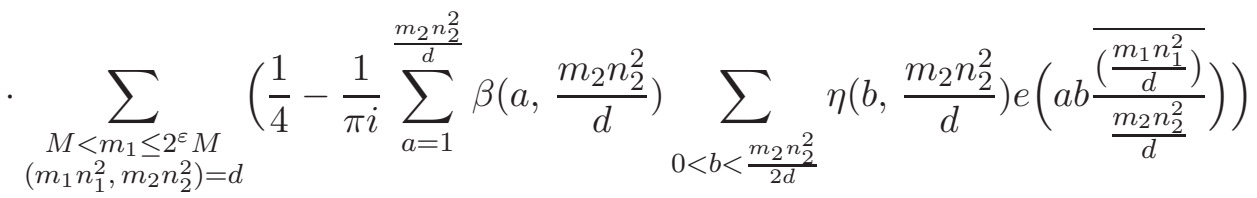

$$
\begin{aligned}
& \ll \sum_{d \leq 8^{\varepsilon} K} \sum_{N<n_{1} \leq 2^{\varepsilon}} \sum_{N}\left|a\left(n_{1}\right) a\left(n_{2}\right)\right| \sum_{M<n_{2} \leq 2^{\varepsilon} N} \frac{1}{m_{2} \leq 2^{\varepsilon} M} \sum_{\substack{M<m_{1} \leq 2^{\varepsilon} M \\
\left(m_{1} n_{1}^{2}, m_{2} n_{2}^{2}\right)=d}} 1 \\
& +\sum_{d \leq 8^{\varepsilon} K} \sum_{N<n_{1} \leq 2^{\varepsilon}} \sum_{N=n_{2} \leq 2^{\varepsilon} N}\left|a\left(n_{1}\right) a\left(n_{2}\right)\right| \sum_{\begin{array}{c}
M<m_{2} \leq 2^{\varepsilon} \\
d \mid m_{2} n_{2}^{2}
\end{array}} \frac{1}{m_{2}} . \\
& \cdot \sum_{a=1}^{\frac{m_{2} n_{2}^{2}}{d}}\left|\beta\left(a, \frac{m_{2} n_{2}^{2}}{d}\right)\right| \sum_{0<b<\frac{m_{2} n_{2}^{2}}{2 d}} \eta\left(b, \frac{m_{2} n_{2}^{2}}{d}\right)\left|\sum_{\substack{M<m_{1} \leq 2^{\varepsilon} M \\
\left(m_{1} n_{1}^{2}, m_{2} n_{2}^{2}\right)=d}} e\left(a b \frac{\overline{\left(\frac{m_{1} n_{1}^{2}}{d}\right)}}{\frac{m_{2} n_{2}^{2}}{d}}\right)\right| \\
& \ll \sum_{N<n_{1} \leq 2^{\varepsilon}} \sum_{N} \sum_{N<n_{2} \leq 2^{\varepsilon} N} \sum_{M<m_{1} \leq 2^{\varepsilon}} \frac{\left|a\left(n_{1}\right) a\left(n_{2}\right)\right|}{m_{2}} \\
& +\sum_{d \leq 8^{\varepsilon} K} \sum_{N<n_{1} \leq 2^{\varepsilon}} \sum_{N}\left|a\left(n_{1}\right) a\left(n_{2}\right)\right| \sum_{\substack{M<m_{2} \leq 2^{\varepsilon} \\
d \mid m_{2} n_{2}^{2}}} \frac{1}{m_{2}} . \\
& \cdot \sum_{a=1}^{\frac{m_{2} n_{2}^{2}}{d}}\left|\beta\left(a, \frac{m_{2} n_{2}^{2}}{d}\right)\right| \sum_{0<b<\frac{m_{2} n_{2}^{2}}{2 d}} \frac{1}{b}\left(a b, \frac{m_{2} n_{2}^{2}}{d}\right)^{\frac{1}{2}}\left(\frac{M N^{2}}{d}\right)^{\frac{1}{2}+\varepsilon} d^{2 \varepsilon} \\
& \ll N^{-2+2 \varepsilon} M^{-1}(M N)^{2}+N^{-2+2 \varepsilon} M^{-1} \sum_{d \leq 8^{\varepsilon} K} \sum_{N<n_{1} \leq 2^{\varepsilon} N} \sum_{N<n_{2} \leq 2^{\varepsilon} N} . \\
& \sum_{\substack{M<m_{2} \leq 2^{\varepsilon} M \\
d \mid m_{2} n_{2}^{2}}}\left(\frac{M N^{2}}{d}\right)^{\frac{1}{2}+\varepsilon} d^{2 \varepsilon} \sum_{a=1}^{\frac{m_{2} n_{2}^{2}}{d}}\left|\beta\left(a, \frac{m_{2} n_{2}^{2}}{d}\right)\right|\left(a, \frac{m_{2} n_{2}^{2}}{d}\right)^{\frac{1}{2}} . \\
& \sum_{0<b<\frac{m_{2} n_{2}^{2}}{2 d}} \frac{1}{b}\left(b, \frac{m_{2} n_{2}^{2}}{d}\right)^{\frac{1}{2}}
\end{aligned}
$$

We have

$$
\begin{aligned}
& \sum_{0<b<\frac{m_{2} n_{2}^{2}}{2 d}} \frac{1}{b}\left(b, \frac{m_{2} n_{2}^{2}}{d}\right)^{\frac{1}{2}}=\sum_{r \mid \frac{m_{2} n_{2}^{2}}{d}} r^{\frac{1}{2}} \sum_{0<b<\frac{m_{2} n_{2}^{2}}{2 d}} \frac{1}{b} \\
& \left(b, \frac{m_{2} n_{2}^{2}}{d}\right)=r
\end{aligned}
$$




$$
\begin{aligned}
& \leq \sum_{r \mid \frac{m_{2} n_{2}^{2}}{d}} r^{\frac{1}{2}} \sum_{\substack{0<b \leq \frac{m_{2} n_{2}^{2}}{2 d} \\
r \mid b}} \frac{1}{b} \\
& \ll \sum_{r \mid \frac{m_{2} n_{2}^{2}}{d}} \frac{1}{r^{\frac{1}{2}}} \log \left(\frac{2 m_{2} n_{2}^{2}}{d}\right) \\
& \ll\left(\frac{m_{2} n_{2}^{2}}{d}\right)^{\frac{\varepsilon}{4}} \\
& \ll\left(\frac{M N^{2}}{d}\right)^{\frac{\varepsilon}{4}}
\end{aligned}
$$

and

$$
\begin{aligned}
& \sum_{a=1}^{\frac{m_{2} n_{2}^{2}}{d}}\left|\beta\left(a, \frac{m_{2} n_{2}^{2}}{d}\right)\right|\left(a, \frac{m_{2} n_{2}^{2}}{d}\right)^{\frac{1}{2}} \\
& \ll \frac{m_{2} n_{2}^{2}}{d}+\sum_{1 \leq a \leq \frac{m_{2} n_{2}^{2}}{2 d}} \frac{\frac{m_{2} n_{2}^{2}}{d}}{a}\left(a, \frac{m_{2} n_{2}^{2}}{d}\right)^{\frac{1}{2}} \\
& +\sum^{\frac{m_{2} n_{2}^{2}}{2 d}<a \leq \frac{m_{2} n_{2}^{2}}{d}-1} \frac{\frac{m_{2} n_{2}^{2}}{d}}{\frac{m_{2} n_{2}^{2}}{d}-a}\left(\frac{m_{2} n_{2}^{2}}{d}-a, \frac{m_{2} n_{2}^{2}}{d}\right)^{\frac{1}{2}} \\
& \ll \frac{m_{2} n_{2}^{2}}{d}+\frac{m_{2} n_{2}^{2}}{d} \quad \sum_{1 \leq a \leq \frac{m_{2} n_{2}^{2}}{2 d}} \frac{1}{a}\left(a, \frac{m_{2} n_{2}^{2}}{d}\right)^{\frac{1}{2}} \\
& \ll\left(\frac{M N^{2}}{d}\right)^{1+\frac{\varepsilon}{4}} .
\end{aligned}
$$

Therefore the contribution of $R_{0}(T ; h, k)$ is

$$
\begin{aligned}
& \ll M N^{2 \varepsilon}+N^{-2+2 \varepsilon} M^{-1} \sum_{d \leq 8^{\varepsilon} K} \sum_{N<n_{1} \leq 2^{\varepsilon} N} \sum_{N<n_{2} \leq 2^{\varepsilon} N} . \\
& \cdot \sum_{M<m_{2} \leq 2^{\varepsilon} M}\left(\frac{M N^{2}}{d}\right)^{\frac{1}{2}+\varepsilon} d^{2 \varepsilon}\left(\frac{M N^{2}}{d}\right)^{1+\frac{\varepsilon}{2}} \\
& \ll M N^{2 \varepsilon}+N^{2 \varepsilon}\left(M N^{2}\right)^{\frac{3}{2}+\frac{3}{2} \varepsilon} \sum_{d \leq 8^{\varepsilon} K} \frac{1}{d^{\frac{3}{2}-\frac{\varepsilon}{2}}} \\
& \ll M N^{2 \varepsilon}+M^{\frac{3}{2}+\frac{3}{2} \varepsilon} N^{3+5 \varepsilon} \\
& \ll \frac{T}{N^{1-8 \varepsilon}} .
\end{aligned}
$$


Combining all of the above, we get

$$
\int_{0}^{\infty} e^{-\frac{t}{T}}\left|\zeta\left(\frac{1}{2}+i t\right)\right|^{2}\left|\sum_{K<k \leq 8^{\varepsilon} K} \frac{b(k)}{k^{i t}}\right|^{2} \ll \frac{T \log ^{2} T}{N^{1-8 \varepsilon}} .
$$

Hence,

$$
\begin{aligned}
& \log T \sum_{r \leq \frac{1}{2 \varepsilon \log 2} \log T+O(1)} \int_{0}^{\infty} e^{-\frac{t}{T}}\left|\zeta\left(\frac{1}{2}+i t\right)\right|^{2} . \\
& \cdot\left|\sum_{2^{\varepsilon r}<m \leq 2^{\varepsilon} \cdot 2^{\varepsilon r}} \frac{1}{m^{\frac{1}{2}+i t}}\right|^{2}\left|\sum_{N<n \leq 2^{\varepsilon} N} \frac{a(n)}{n^{2 i t}}\right|^{2} d t \ll \frac{T \log ^{4} T}{N^{1-8 \varepsilon}} .
\end{aligned}
$$

So far the proof of Proposition 3 is finished.

Proof of Proposition 2. We observe that the measure of the set of all $t$ such that $\frac{T}{2} \leq t \leq T$ and $2 t \in U_{4}$ is $\ll T^{11 \varepsilon}(\log T)^{10}$. We suppose firstly that $k=2 m$ with positive integer $m$. By Proposition 1 , Lemmas 8,9 and 11 ,

$$
\begin{aligned}
& \int_{\frac{T}{2}}^{T}\left|\zeta\left(\frac{1}{2}+i t\right)\right|^{4}|\zeta(1+2 i t)|^{-2 m} d t \\
& =\int_{\frac{T}{2} \leq t \leq T, 2 t \notin U_{4}}\left|\zeta\left(\frac{1}{2}+i t\right)\right|^{4}|\zeta(1+2 i t)|^{-2 m} d t \\
& +\int_{\frac{T}{2} \leq t \leq T, 2 t \in U_{4}}\left|\zeta\left(\frac{1}{2}+i t\right)\right|^{4}|\zeta(1+2 i t)|^{-2 m} d t \\
& \ll \int_{\frac{T}{2} \leq t \leq T, 2 t \notin U_{4}}\left|\zeta\left(\frac{1}{2}+i t\right)\right|^{4}\left(\left|\sum_{l \leq X} \frac{\mu(l)}{l^{1+2 i t}} \cdot e^{-\frac{l}{X}}\right|^{2 m}+O(1)\right) d t+O\left(T \log ^{4} T\right) \\
& \ll \int_{\frac{T}{2}}^{T}\left|\zeta\left(\frac{1}{2}+i t\right)\right|^{4}\left|\sum_{l \leq X} \frac{\mu(l)}{l^{1+2 i t}} \cdot e^{-\frac{l}{X}}\right|^{2 m} d t+O\left(T \log ^{4} T\right) \\
& =\int_{\frac{T}{2}}^{T}\left|\zeta\left(\frac{1}{2}+i t\right)\right|^{4}\left|\sum_{n \leq X^{m}} \frac{a(n)}{n^{2 i t}}\right|^{2} d t+O\left(T \log ^{4} T\right)
\end{aligned}
$$

where

$$
a(n)=\frac{1}{n} \sum_{l_{1} \cdots l_{m}=n} \mu\left(l_{1}\right) \cdots \mu\left(l_{m}\right) \exp \left(-\frac{\left(l_{1}+\cdots+l_{m}\right)}{X}\right),
$$

$U_{4}$ is defined as in (3.2), $X$ is defined as in (3.8). We can see

$$
X^{m}=\exp \left(\frac{2 m}{\varepsilon}(\log T)^{\frac{3}{4}}\right) \ll T^{\frac{1}{16}-2 \varepsilon}
$$


and

$$
a(n)=O\left(n^{-1+\varepsilon}\right) .
$$

By Cauchy's inequality,

$$
\begin{aligned}
\left|\sum_{n \leq X^{m}} \frac{a(n)}{n^{2 i t}}\right|^{2} & =\left|\sum_{s \leq \frac{m \log X}{\varepsilon \log 2}-1} \frac{1}{2^{\frac{\varepsilon s}{4}}} \cdot 2^{\frac{\varepsilon s}{4}} \sum_{2^{\varepsilon s}<n \leq 2^{\varepsilon} 2^{\varepsilon s}} \frac{a(n)}{n^{2 i t}}\right|^{2} \\
& \leq\left.\left.\sum_{s \leq \frac{m \log X}{\varepsilon \log 2}-1} \frac{1}{2^{\frac{\varepsilon s}{2}}} \sum_{s \leq \frac{m \log X}{\varepsilon \log 2}-1} 2^{\frac{\varepsilon s}{2}}\right|_{2^{\varepsilon s}<n \leq 2^{\varepsilon} 2^{\varepsilon s}} \frac{a(n)}{n^{2 i t}}\right|^{2} \\
& \left.\ll \sum_{s \leq \frac{m \log X}{\varepsilon \log 2}-1} 2^{\frac{\varepsilon s}{2}} \sum_{2^{\varepsilon s}<n \leq 2^{\varepsilon} 2^{\varepsilon s}} \frac{a(n)}{n^{2 i t}}\right|^{2} .
\end{aligned}
$$

Hence, Proposition 3 yields

$$
\begin{aligned}
& \int_{\frac{T}{2}}^{T}\left|\zeta\left(\frac{1}{2}+i t\right)\right|^{4}\left|\sum_{n \leq X^{m}} \frac{a(n)}{n^{2 i t}}\right|^{2} d t \\
& \ll \sum_{s \leq \frac{m \log X}{\varepsilon \log 2}-1} 2^{\frac{\varepsilon s}{2}} \int_{\frac{T}{2}}^{T}\left|\zeta\left(\frac{1}{2}+i t\right)\right|^{4}\left|\sum_{2^{\varepsilon s}<n \leq 2^{\varepsilon} 2^{\varepsilon s}} \frac{a(n)}{n^{2 i t}}\right|^{2} \\
& \ll \sum_{s \leq \frac{m \log X}{\varepsilon \log 2}-1} 2^{\frac{\varepsilon s}{2}} \cdot \frac{T \log ^{4} T}{2^{\varepsilon s(1-8 \varepsilon)}} \\
& \ll T \log ^{4} T .
\end{aligned}
$$

Thus,

$$
\int_{\frac{T}{2}}^{T}\left|\zeta\left(\frac{1}{2}+i t\right)\right|^{4}|\zeta(1+2 i t)|^{-2 m} d t \ll T \log ^{4} T .
$$

Therefore

$$
\int_{1}^{T}\left|\zeta\left(\frac{1}{2}+i t\right)\right|^{4}|\zeta(1+2 i t)|^{-2 m} d t \ll T \log ^{4} T
$$

For the general $k>0$, we have an even integer $2 m$ such that $k<2 m$.

By Hölder's inequality,

$$
\begin{aligned}
& \int_{1}^{T}\left|\zeta\left(\frac{1}{2}+i t\right)\right|^{4}|\zeta(1+2 i t)|^{-k} d t \\
= & \int_{1}^{T}\left|\zeta\left(\frac{1}{2}+i t\right)\right|^{4 \cdot \frac{2 m-k}{2 m}} \cdot\left|\zeta\left(\frac{1}{2}+i t\right)\right|^{4 \cdot \frac{k}{2 m}}|\zeta(1+2 i t)|^{-k} d t \\
\leq & \left(\int_{1}^{T}\left|\zeta\left(\frac{1}{2}+i t\right)\right|^{4 \cdot \frac{2 m-k}{2 m} \cdot \frac{2 m}{2 m-k}} d t\right)^{\frac{2 m-k}{2 m}} \cdot \\
& \cdot\left(\int_{1}^{T}\left|\zeta\left(\frac{1}{2}+i t\right)\right|^{4 \cdot \frac{k}{2 m} \cdot \frac{2 m}{k}}|\zeta(1+2 i t)|^{-k \cdot \frac{2 m}{k}} d t\right)^{\frac{k}{2 m}}
\end{aligned}
$$




$$
\begin{aligned}
& =\left(\int_{1}^{T}\left|\zeta\left(\frac{1}{2}+i t\right)\right|^{4} d t\right)^{\frac{2 m-k}{2 m}}\left(\int_{1}^{T}\left|\zeta\left(\frac{1}{2}+i t\right)\right|^{4}|\zeta(1+2 i t)|^{-2 m} d t\right)^{\frac{k}{2 m}} \\
& \ll T \log ^{4} T .
\end{aligned}
$$

So far the proof of Proposition 2 is finished.

\section{The proof of Theorem}

We shall apply Lemma 5. For $\operatorname{Re}(s)>1$, let

$$
f(s)=\sum_{n=1}^{\infty} \frac{d^{2}(n)}{n^{s}} \text {. }
$$

By Lemma 6,

$$
f(s)=\frac{\zeta^{4}(s)}{\zeta(2 s)} .
$$

It is easy to see that $\psi(n)=n^{\varepsilon}$ which is non-decreasing. As $\sigma \rightarrow 1^{+}$,

$$
\sum_{n=1}^{\infty} \frac{d^{2}(n)}{n^{\sigma}}=\frac{\zeta^{4}(\sigma)}{\zeta(2 \sigma)}=O\left(\frac{1}{(\sigma-1)^{4}}\right) .
$$

Let $c=1+\varepsilon, Y=[x]+\frac{1}{2}, T=x^{\frac{3}{4}}$. Then

$$
\begin{aligned}
\sum_{n \leq x} d^{2}(n) & =\sum_{n<Y} d^{2}(n)+O_{\varepsilon}\left(x^{\varepsilon}\right) \\
& =\frac{1}{2 \pi i} \int_{1+\varepsilon-i T}^{1+\varepsilon+i T} \frac{\zeta^{4}(s)}{\zeta(2 s)} \cdot \frac{Y^{s}}{s} d s+O_{\varepsilon}\left(x^{\frac{1}{4}+2 \varepsilon}\right) .
\end{aligned}
$$

We move the line of integration to $\operatorname{Re}(s)=\frac{1}{2}$. The residue of $\frac{\zeta^{4}(s)}{\zeta(2 s)} \cdot \frac{Y^{s}}{s}$ at $s=1$ is

$$
Y P(\log Y)=x P(\log x)+O\left(x^{\varepsilon}\right) .
$$

By Lemmas 8 and 10,

$$
\frac{1}{2 \pi i} \int_{\frac{1}{2}+i T}^{1+\varepsilon+i T} \frac{\zeta^{4}(s)}{\zeta(2 s)} \cdot \frac{Y^{s}}{s} d s \ll \max _{\frac{1}{2} \leq \sigma \leq 1+\varepsilon} T^{\frac{4}{3}(1-\sigma)+4 \varepsilon} \log T \cdot \frac{x^{\sigma}}{T} \ll x^{\frac{1}{4}+4 \varepsilon} .
$$

In the same way,

$$
\frac{1}{2 \pi i} \int_{\frac{1}{2}-i T}^{1+\varepsilon-i T} \frac{\zeta^{4}(s)}{\zeta(2 s)} \cdot \frac{Y^{s}}{s} d s \ll x^{\frac{1}{4}+4 \varepsilon}
$$

Hence,

$$
E(x)=\frac{1}{2 \pi i} \int_{\frac{1}{2}-i T}^{\frac{1}{2}+i T} \frac{\zeta^{4}(s)}{\zeta(2 s)} \cdot \frac{Y^{s}}{s} d s+O\left(x^{\frac{1}{4}+4 \varepsilon}\right) .
$$


It follows from Proposition 2 that,

$$
\begin{aligned}
E(x) & \ll x^{\frac{1}{2}} \sum_{k \leq \frac{\log T}{\log 2}} \int_{2^{k-1}}^{2^{k}} \frac{\left|\zeta\left(\frac{1}{2}+i t\right)\right|^{4}}{|\zeta(1+2 i t)|} \frac{d t}{t}+O\left(x^{\frac{1}{2}}\right) \\
& \ll x^{\frac{1}{2}} \sum_{k \leq \frac{\log T}{\log 2}} \frac{1}{2^{k}} \int_{1}^{2^{k}} \frac{\left|\zeta\left(\frac{1}{2}+i t\right)\right|^{4}}{|\zeta(1+2 i t)|} d t+O\left(x^{\frac{1}{2}}\right) \\
& \ll x^{\frac{1}{2}} \sum_{k \leq \frac{\log T}{\log 2}} \frac{1}{2^{k}} \cdot 2^{k} k^{4} \\
& \ll x^{\frac{1}{2}} \log ^{5} x .
\end{aligned}
$$

Thus, the proof of the Theorem is complete.

\section{Some remarks}

By the method of this paper, we can prove that if $k$ is any given positive number, $a$ is a given positive integer, then

$$
\int_{1}^{T} \frac{\left|\zeta\left(\frac{1}{2}+i t\right)\right|^{4}}{|\zeta(1+a i t)|^{k}} d t \ll_{k, a} T \log ^{4} T .
$$

We note that if $\operatorname{Re}(s)>1$,

$$
\sum_{n=1}^{\infty} \frac{d\left(n^{3}\right)}{n^{s}}=\frac{\zeta^{4}(s)}{\zeta^{3}(2 s)} \cdot G_{1}(s),
$$

where

$$
G_{1}(s)=\prod_{p} \frac{\left(1+\frac{2}{p^{s}}\right)}{\left(1-\frac{1}{p^{s}}\right)\left(1+\frac{1}{p^{s}}\right)^{3}},
$$

$G_{1}(s)$ is absolutely convergent for $\operatorname{Re}(s)>\frac{1}{3}$. One can see page 95 in [2]. Using the method similar to that in this paper, we can prove the following proposition.

Proposition 4. We have

$$
\sum_{n \leq x} d\left(n^{3}\right)=x P_{1}(\log x)+O\left(x^{\frac{1}{2}}(\log x)^{5}\right),
$$

where $P_{1}(y)$ is a suitable cubic polynomial in $y$.

In 2006, M. Z. Garaev, F. Luca and W. G. Nowak[2] proved that as $x \rightarrow \infty$, if $y=y(x)$ satisfies

$$
\frac{y}{x^{\frac{1}{2}} \log x \log \log x} \rightarrow \infty
$$


then

$$
\sum_{x<n \leq x+y} d^{2}(n) \sim \frac{6}{\pi^{2}} y(\log x)^{3},
$$

and that as $x \rightarrow \infty$, if $y=y(x)$ satisfies

$$
\frac{y}{x^{\frac{1}{2}} \log x(\log \log x)^{3}} \rightarrow \infty,
$$

then

$$
\sum_{x<n \leq x+y} d\left(n^{3}\right) \sim B_{0} y(\log x)^{3}
$$

where $B_{0}$ is a positive constant.

Combining the method of this paper with that of [2], we can prove the following proposition.

Proposition 5. As $x \rightarrow \infty$, if $y=y(x)$ satisfies

$$
\frac{y}{x^{\frac{1}{2}} \log x} \rightarrow \infty
$$

then

$$
\sum_{x<n \leq x+y} d^{2}(n) \sim \frac{6}{\pi^{2}} y(\log x)^{3}
$$

and

$$
\sum_{x<n \leq x+y} d\left(n^{3}\right) \sim B_{0} y(\log x)^{3} .
$$

Let $r(n)$ be the number of representations of $n$ as the sum of two squares. In 2004, M. Kühleitner and W. G. Nowak[6] proved that

$$
\sum_{n \leq x} r^{2}(n)=4 x \log x+B_{1} x+O\left(x^{\frac{1}{2}}(\log x)^{3} \log \log x\right),
$$

where $B_{1}$ is a positive constant, and that

$$
\sum_{n \leq x} r\left(n^{3}\right)=A_{2} x \log x+B_{2} x+O\left(x^{\frac{1}{2}}(\log x)^{3}(\log \log x)^{2}\right),
$$

where $A_{2}, B_{2}$ are positive constants.

Let $\mathbf{K}=\mathbb{Q}(i), \zeta_{\mathbf{K}}(s)$ be the Dedekind $\zeta$ function in the field $\mathbf{K}$. If $\operatorname{Re}(s)>1$,

$$
\begin{aligned}
& \sum_{n=1}^{\infty} \frac{r^{2}(n)}{n^{s}}=\frac{16 \zeta_{\mathbf{K}}^{2}(s)}{\left(1+2^{-s}\right) \zeta(2 s)}, \\
& \sum_{n=1}^{\infty} \frac{r\left(n^{3}\right)}{n^{s}}=\frac{\zeta_{\mathbf{K}}^{2}(s)}{\zeta(2 s) \zeta_{\mathbf{K}}(2 s)} \cdot G_{2}(s),
\end{aligned}
$$


where $G_{2}(s)$ is holomorphic and bounded for $\operatorname{Re}(s)>\frac{1}{3}+\varepsilon$. One can see (4.1) and (4.4) in [6].

If the result of Iwaniec[4] could be generalized to $\zeta_{\mathbf{K}}(s)$, then the error terms in (6.2) and (6.3) could be improved to $O\left(x^{\frac{1}{2}}(\log x)^{3}\right)$. Furthermore, the sums studied in $[2]$

$$
\sum_{x<n \leq x+y} r^{2}(n), \quad \sum_{x<n \leq x+y} r\left(n^{3}\right), \quad \sum_{x<n \leq x+y} d(n) r(n)
$$

could also be improved correspondingly.

\section{Acknowledgements}

The authors would like to thank the referee for his/her nice comments and suggestions. Chaohua Jia is supported by the National Natural Science Foundation of China (Grant No. 11371344) and the National Key Basic Research Program of China (Project No. 2013CB834202).

\section{References}

[1] T. Estermann, On the representation of a number as the sum of two products, Proc. London Math. Soc.(2) 31(1930), 123-133.

[2] M. Z. Garaev, F. Luca and W. G. Nowak, Short interval asymptotics for a class of arithmetic functions, Acta Math. Hungar. 113(1-2)(2006), 85-99.

[3] M. N. Huxley, On the difference between consecutive primes, Invent. Math. 15(1972), 155-164.

[4] H. Iwaniec, On mean values for Dirichlet's polynomials and the Riemann zeta-function, J. London Math. Soc.(2) 22(1980), 39-45.

[5] M. Kühleitner and W. G. Nowak, An omega theorem for a class of arithmetic functions, Math. Nachr. 165(1994), 79-98. 
[6] M. Kühleitner and W. G. Nowak, The average number of solutions of the Diophantine equation $U^{2}+V^{2}=W^{3}$ and related arithmetic functions, Acta Math. Hungar. 104(3)(2004), 225-240.

[7] H. Maier and A. Sankaranarayanan, On the error term of an asymptotic formula of Ramanujan, Acta Arith. 117(2005), no.2, 171-180.

[8] K. Ramachandra and A. Sankaranarayanan, On an asymptotic formula of Srinivasa Ramanujan, Acta Arith. 109(2003), no.4, 349-357.

[9] S. Ramanujan, Some formulae in the analytic theory of numbers, Messenger of Math. 45(1916), 81-84.

[10] D. Suryanarayana and R. Sitaramachandra Rao, On an asymptotic formula of Ramanujan, Math. Scand. 32(1973), 258-264.

[11] E. C. Titchmarsh, The Theory of Functions, 2nd edition, Oxford University Press, London, 1939.

[12] E. C. Titchmarsh, The Theory of the Riemann Zeta-Function, 2nd edititon, Clarendon Press, Oxford, 1986.

[13] B. M. Wilson, Proofs of some formulae enunciated by Ramanujan, Proc. London Math. Soc.(2) 21(1922), 235-255.

Chaohua Jia

Institute of Mathematics, Academia Sinica, Beijing 100190, P. R. China Hua Loo-Keng Key Laboratory of Mathematics, Chinese Academy of Sciences, Beijing 100190, P. R. China

E-mail: jiach@math.ac.cn

Ayyadurai Sankaranarayanan

School of Mathematics, Tata Institute of Fundamental Research, Homi Bhabha Road, Mumbai 400005, India

E-mail: sank@math.tifr.res.in 